\title{
Colicin-Mediated Transport of DNA through the Iron Transporter FepA
}

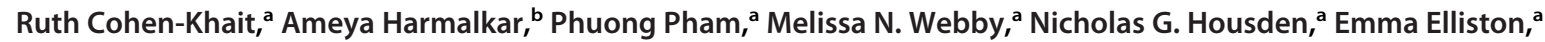

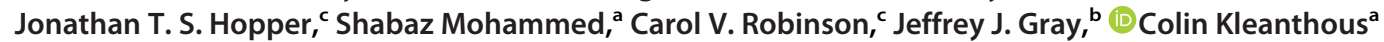

aDepartment of Biochemistry, University of Oxford, Oxford, United Kingdom

${ }^{b}$ Chemical \& Biomolecular Engineering, Johns Hopkins University, Baltimore, Maryland, USA

'Department of Chemistry, University of Oxford, Oxford, United Kingdom

Ruth Cohen-Khait and Ameya Harmalkar contributed equally.

ABSTRACT Colicins are protein antibiotics deployed by Escherichia coli to eliminate competing strains. Colicins frequently exploit outer membrane (OM) nutrient transporters to penetrate the selectively permeable bacterial cell envelope. Here, by applying live-cell fluorescence imaging, we were able to monitor the entry of the pore-forming toxin colicin B (ColB) into E. coli and localize it within the periplasm. We further demonstrate that single-stranded DNA coupled to ColB can also be transported to the periplasm, emphasizing that the import routes of colicins can be exploited to carry large cargo molecules into bacteria. Moreover, we characterize the molecular mechanism of ColB association with its OM receptor FepA by applying a combination of photoactivated cross-linking, mass spectrometry, and structural modeling. We demonstrate that complex formation is coincident with large-scale conformational changes in the colicin. Thereafter, active transport of ColB through FepA involves the colicin taking the place of the $\mathrm{N}$-terminal half of the plug domain that normally occludes this iron transporter.

IMPORTANCE Decades of excessive use of readily available antibiotics has generated a global problem of antibiotic resistance and, hence, an urgent need for novel antibiotic solutions. Bacteriocins are protein-based antibiotics produced by bacteria to eliminate closely related competing bacterial strains. Bacteriocin toxins have evolved to bypass the complex cell envelope in order to kill bacterial cells. Here, we uncover the cellular penetration mechanism of a well-known but poorly understood bacteriocin called colicin B that is active against Escherichia coli. Moreover, we demonstrate that the colicin B-import pathway can be exploited to deliver conjugated DNA cargo into bacterial cells. Our work leads to a better understanding of the way bacteriocins, as potential alternative antibiotics, execute their mode of action as well as highlighting how they might even be exploited in the genomic manipulation of Gramnegative bacteria.

KEYWORDS membrane transport, bacteriocins, antibiotic resistance, conformational changes, Rosetta flexible backbone, DNA delivery

acteria are the most common and diverse form of life on earth. The remarkable abundance of different bacterial strains and species capable of surviving in almost any environment frequently leads to competition for space and resources (1). Competition for scarce nutrients has led to the evolution of nutrient uptake systems, such as the secretion of siderophores to chelate bioavailable iron, with the iron-siderophore complex captured by high-affinity receptors and actively transported across the cell envelope (2). Competing bacteria also deploy weapons in the form of enzymes
Citation Cohen-Khait R, Harmalkar A, Pham P, Webby MN, Housden NG, Elliston E, Hopper JTS, Mohammed S, Robinson CV, Gray JJ, Kleanthous C. 2021. Colicin-mediated transport of DNA through the iron transporter FepA. mBio 12:e01787-21. https://doi.org/10.1128/ mBio.01787-21.

Editor Mark J. Bailey, CEH-Oxford

Copyright $\odot 2021$ Cohen-Khait et al. This is an open-access article distributed under the terms of the Creative Commons Attribution 4.0 International license.

Address correspondence to Jeffrey J. Gray, jgray@jhu.edu, or Colin Kleanthous, colin.kleanthous@bioch.ox.ac.uk.

Received 15 June 2021

Accepted 23 August 2021

Published 21 September 2021 
targeting either components of the cell wall or nucleic acids (3) or depolarizing pores that disrupt the electrochemical potential across the inner membrane (4). Elimination of competing bacteria while kin bacteria are unharmed is achieved through the coexpression of toxin-specific immunity proteins that render the toxin inactive within producing strains (5). Cytotoxic proteins can be delivered either in a contact-dependent manner, targeting neighboring cells relying on the assembly of supramolecular machineries (6), or through secretion into the milieu as exemplified by bacteriocins (7).

Colicins, the bacteriocins of $E$. coli, have been extensively studied, with over 20 different examples described (8). Once released, colicins breach the envelope of their target cell to elicit their cytotoxic activity (9). The cell envelope of Gram-negative bacteria is comprised of an asymmetric outer membrane (OM) with an outer leaflet comprised of lipopolysaccharide and a phospholipid inner leaflet, providing a robust layer of defense surrounding the energized inner membrane (IM) and the intervening periplasm (10). Colicins are large ( 29 to $75 \mathrm{kDa}$ ) proteins that cannot diffuse through the cell envelope of their target cell (11) and must find a route across the OM (12). Unlike the proton-motive force (PMF) of the IM, the OM is not directly energized, and energydependent processes at the OM such as protein import are coupled to the IM through trans-periplasmic complexes. The Tol-Pal system, composed of the TolQ-TolR-TolA complex in the IM, TolB in the periplasm, and Pal anchored to the inner leaflet of the OM, stabilizes the OM during cell division (13). The structurally related Ton system, composed of the TonB-ExbB-ExbD complex in the IM, powers active transport of nutrients such as siderophores through specialized TonB-dependent receptors in the OM (14). Both the Tol-Pal and the Ton systems are exploited by colicins to energize their translocation across the cell envelope.

Colicins typically contain three structural domains, a central receptor (R)-binding domain, which anchors the toxin to the cell surface, an N-terminal translocation (T) domain implicated in OM translocation via the Tol-Pal, or a Ton system of a C-terminal cytotoxic domain. Colicin $\mathrm{B}(\mathrm{ColB})$ is a pore-forming toxin that was one of the earliest colicins to be described (15). However, little is known about the cellular translocation process of ColB beyond its dependence on the OM ferric enterobactin transporter FepA and the Ton system (16). No additional OM proteins have been identified for ColB toxicity, which may explain why, unlike most other colicins, ColB is composed of only two functional domains: an N-terminal domain that serves as both a receptorbinding domain and a translocation domain (ColB-RT) and a pore-forming, C-terminal cytotoxic domain (17). The ColB receptor FepA is a 22-stranded $\beta$-barrel TonB-dependent transporter (TBDT) with an N-terminal plug domain blocking its lumen (18-20).

Here, we elucidate the mechanism by which ColB interacts with its receptor FepA and its active transport across the OM. Applying live-cell fluorescence microscopy, we visualize the translocation of the ColB-RT domain to the periplasm of E. coli, demonstrating that translocation requires FepA at the OM and depends on colicin's TonB box. We applied a combined approach of in vitro and in vivo photoactivated cross-linking, mass spectrometry, and structural modeling with Rosetta to monitor the key stages in the ColB-FepA association process. We also demonstrate that the import route of ColB can be used to import large macromolecules, in this instance single-stranded DNA (ssDNA), into bacteria.

\section{RESULTS}

ColB can transport ssDNA into $E$. coli via FepA. Previous work has demonstrated that Pseudomonas aeruginosa-specific pyocins are capable of transporting fluorophores into target cells $(21,22)$. Whether this is also possible for colicins and $E$. coli has yet to be determined. To address this question, the ColB-RT domain (residues 1 to 341) was labeled with Alexa Fluor 488 through modification of a cysteine residue at its $C$ terminus and entry into $E$. coli investigated by fluorescence microscopy (Fig. 1A). Addition of trypsin was used to distinguish between ColB bound at the outer membrane and that which had translocated into the cells. Deletion of the ColB TonB box (residues 17 to 


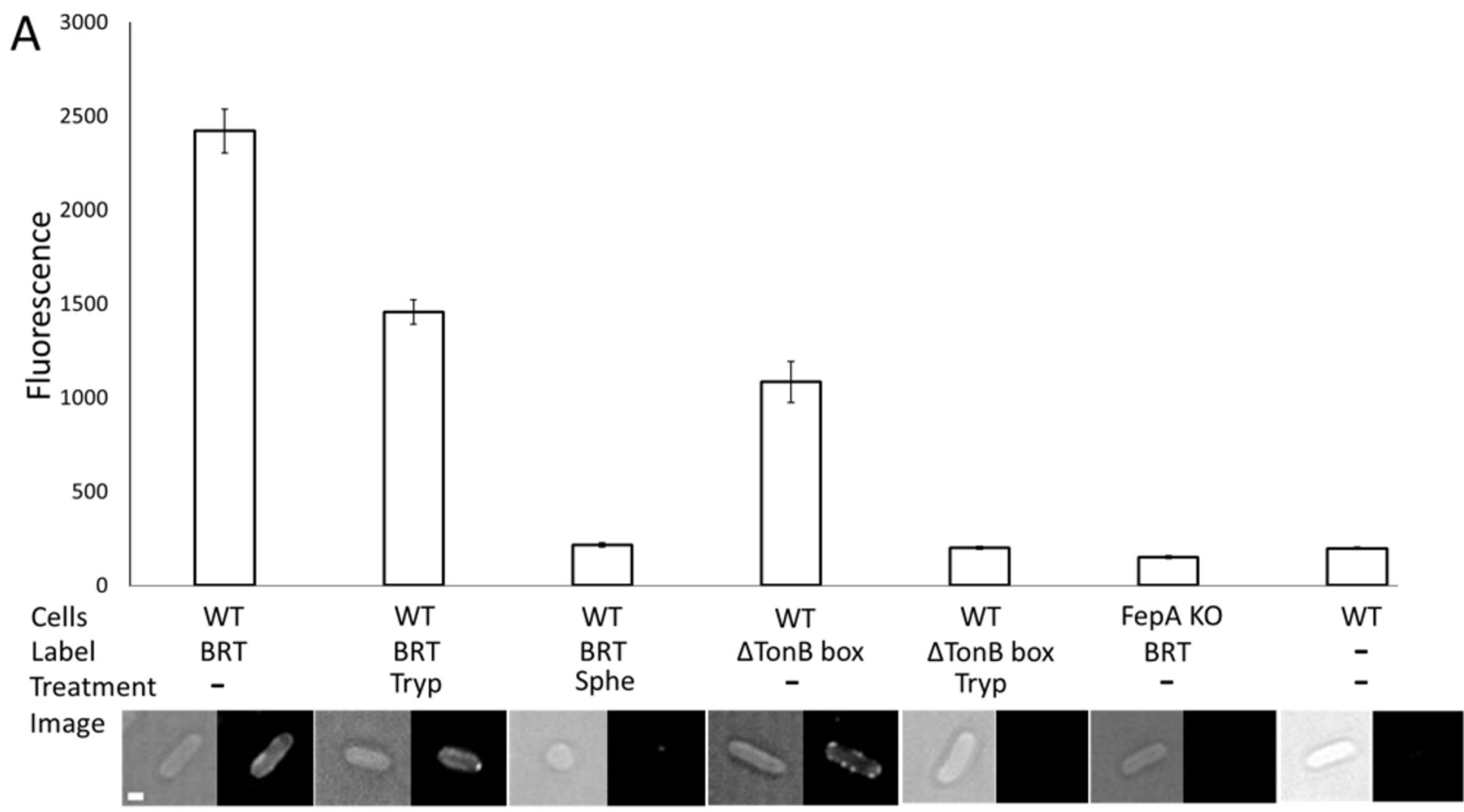

B

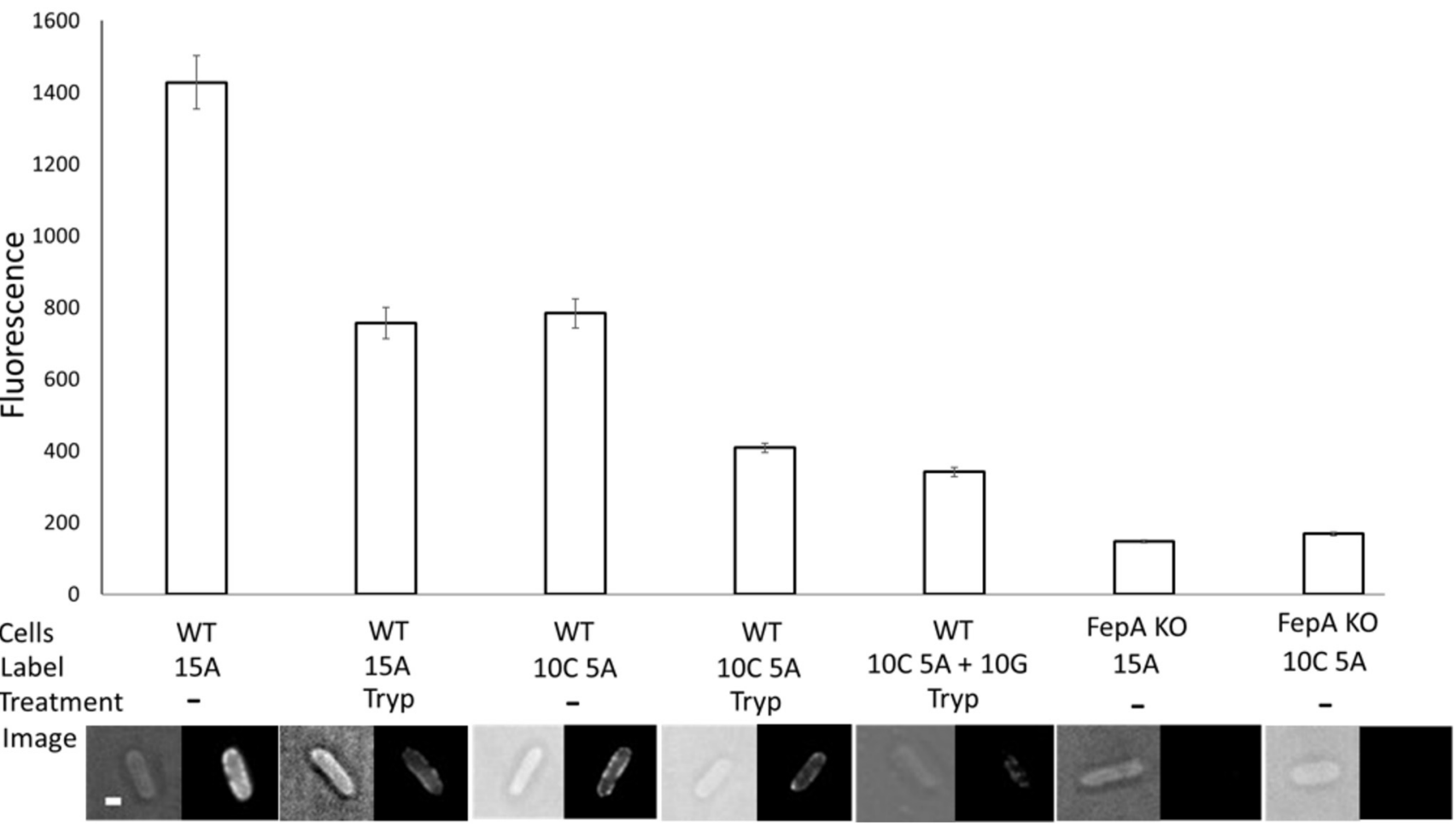

FIG 1 ssDNA follows the ColB-RT translocation path into E. coli cells. (A) Translocation of ColB-RT-Alexa 488 (BRT) or ColB-RT $\Delta$ TonB box-Alexa 488 ( $\Delta$ TonB box) constructs into E. coli MG1655 cells (WT) or E. coli BW25113 $\Delta$ FepA (FepA knockout [KO]) cells grown in minimal medium to mid-log growth phase $\left(\mathrm{OD}_{600}, \sim 0.35\right)$. OM translocation was defined as fluorescent signal resistant to trypsin treatment (Tryp). Cytoplasmic localization was defined as fluorescent signal remaining after spheroplasting the cells, which results in the removal of the OM and the periplasmic peptidoglycan layer (Sphe). The averaged fluorescence intensities were calculated from at least 120 cells (30 cells $\times 4$ biological repeats), and standard error bars of each treatment are shown. Representative cellular images are below each treatment. Scale bar, $1 \mu \mathrm{m}$. (B) Translocation of ColB-RT-DNA-fused constructs were ColB-RT-A ${ }_{15}$ Alexa 488 (15A), ColB-RT $A_{5} C_{10}$ Alexa 488 (10C 5A), and ColB-RT $A_{5} C_{10}$ Alexa $488+G_{10}\left(A_{5} C_{10}+G_{10}\right)$. 
21) resulted in the complete loss of colicin's ability to translocate, consistent with its Ton dependence; however, it maintained its ability to bind FepA (Fig. 1A). No binding was detected in a fepA deletion strain (Fig. 1A). Pore-forming colicins translocate to the periplasm to elicit cell death. Consistent with the periplasm being the final destination of ColB, the fluorescent signal of ColB-RT was lost upon spheroplasting cells (Fig. 1A). Having established that ColB-RT could transport a fluorophore to the periplasm, we then investigated whether the colicin could be used to deliver macromolecules into the cell, specifically ssDNA. The C-terminal cysteine of ColB-RT was modified with fluorescently labeled ssDNA $\left(A_{15}\right.$ or $A_{5} C_{10}$ ). DNase treatment of the ssDNA-labeled ColB-RT released the fluorophore (see Fig. S5 in the supplemental material); hence, DNase treatment could be used to distinguish between surface-bound ColB-RT and that which had translocated to the periplasm. As with fluorescently labeled ColB-RT, ColB-RT conjugated to fluorescently labeled DNA translocated to the periplasm in an FepA-dependent manner (Fig. 1B). Addition of a $G_{10}$ oligonucleotide to ColB-RT conjugated to $A_{5} C_{10}$ decreased the fluorescence signal detected in the periplasm (Fig. 1B), suggesting either that dsDNA is transported poorly or that dissociation of the second DNA strand slows the translocation process.

Receptor FepA binding induces large-scale conformational changes in ColB. Many colicins bind multiple OM proteins or even multiple copies of the same OM protein (9). To address the question of whether additional proteins or copies of FepA were required for ColB transport, ColB complexes were assembled on the surface of ColBsensitive $E$. coli, detergent extracted, and purified by nickel affinity, followed by size exclusion chromatography. Native mass spectrometry of this isolated complex revealed the FepA-ColB complex to have a 1:1 stoichiometry (Fig. S1), consistent with ColB binding and translocating through a single copy of FepA. No structures exist for the ColB-FepA complex. Hence, to understand how colicin associates with this TBDT, we resorted to a Rosetta docking algorithm (23), exploiting available PDB structures of unbound ColB (24) (PDB entry 1RH1) and FepA (18) (PDB entry 1FEP). The structures were initially positioned with the FepA extracellular loops facing the predicted ColB receptor-binding loops (25) (Fig. S2). This calculation revealed a clear energy funnel (Fig. 2D) for an encounter complex (EC) structure (Fig. 2A). As an independent test of the Rosetta model predictions we used pBPA cross-linking. We introduced p-benzoyl-Lphenylalanine (pBPA) mutations into ColB-RT surface loops, previously highlighted as potential FepA binding sites (25) (Fig. S2). Exposure to UV (365 nm) results in pBPA nonspecific cross-linking into $\mathrm{C}-\mathrm{H}$ bonds within $\sim 4 \AA$ (26). Photoactivated cross-linking experiments were performed both in vitro, using an OM protein fraction as a FepA source, and in vivo, using live $E$. coli cells. We identified cross-links by SDS-PAGE and further analyzed these by liquid chromatography-tandem mass spectrometry (LC-MS/ MS), as previously described by White et al. (21). We identified three cross-links in vitro, two of which (ColB residues D202X and R205X with FepA residues P642 and K639, respectively) validated the EC computed by Rosetta (Fig. 2A and Fig. S3A and S4A to C), the success of the computational prediction is a likely consequence of recent progress in the Rosetta docking energy function $(23,27)$. However, a third cross-link, ColB residue Q55X with FepA residue S652, could not be explained by the computed EC. ColB Q55 is in close proximity ( $~ 8 \AA)$ to ColB D202 and R205 in the ColB PDB structure (PDB entry $1 \mathrm{RH} 1$ ), yet its mapped FepA cross-link appears $28 \AA$ apart from the mapped cross-link of ColB 205 (Fig. 2B). This disagreement was suggestive of a conformational change accompanying formation of the complex. Hence, to improve the structural model of the ColB-FepA complex, we simulated the N-terminal portion of ColB (residues 1 to 55) as a floppy tail, allowing it to sample its environment freely (23). The resulting model of the stable complex (SC) now explains all three in vitro-observed cross-links (Fig. 1C) and is more energetically favorable than the initially calculated EC (Fig. 2D and Movie S1). The calculated SC also brings the ColB TonB box (residues 17 to 21) closer to the FepA lumen (Fig. 2C). In conclusion, using a combination of photoactivated crosslinking and Rosetta-based docking simulations, we have uncovered that ColB associates 


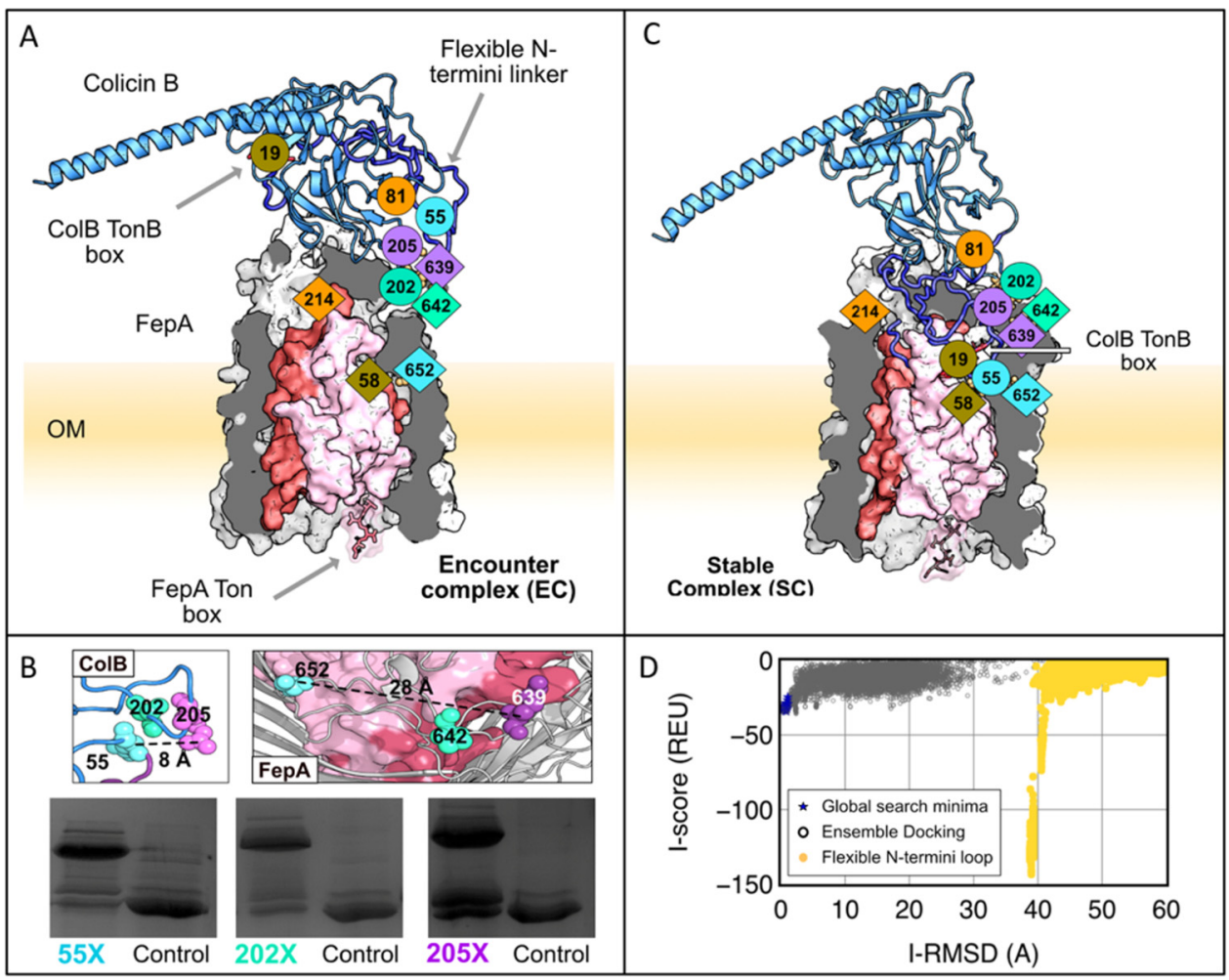

FIG 2 Structural insights on the ColB-FepA complex by pBPA cross-linking and Rosetta-based structural modeling. (A) Initial encounter complex (EC) modeled with moderate to little backbone flexibility (under $5 \AA$ root mean square deviations [RMSD]). ColB (blue) and FepA (gray) form this encounter complex with in vitro cross-links, FepA-K639 and ColB-D202 (teal), and FepA-P642 and ColB-R205 (purple), which lie in proximity in the model. The last in vitro cross-link pair, FepA-S652 and ColB-Q55 (cyan), and the two in vivo cross-links, FepA-T58 and ColB-M19 (olive) and FepA A214 and ColB-G81 (orange), are not satisfied in this structure. (B) Mapped in vitro cross-linking sites on the ColB and FepA PDB structures (1RH1 and 1FEP, respectively). Cropped relevant cross-link gels. Self-cross-linking control to the right of each lane (full in vitro cross-linking image is in Fig. S3A). (C) Fully assembled spontaneously formed stable complex (SC) modeled with the Rosetta FlopyTail algorithm (48) simulating the partially unstructured ColB 1-55 as a floppy tail. (D) Rosetta interface score ( $y$ axis) versus interface RMSD ( $x$ axis) for output structures identified by local docking (ReplicaDock2) of ColB to FepA. RMSD is measured relative to the lowest-scoring global docking structure. There is a deep minimum resulting from the arrangement of the flexible $\mathrm{N}$-linker for the FloppyTail models. Measurements corresponding to panel $\mathrm{A}$ are in navy blue, measurements corresponding to panel $\mathrm{C}$ are in yellow.

with its receptor/translocator FepA through an initial encounter complex that then rearranges to the final stable complex, which prepares the toxin for import.

ColB exploits FepA for its active translocation into the cell. The route taken by ColB during FepA-dependent translocation is unknown. Here, we show how the partially unstructured flexible $\mathrm{N}$-terminal tail of ColB (residues 1 to 55) occupies the channel generated by the TonB-dependent unfolding of the N-terminal half of the FepA plug domain (Fig. 3). While complex formation (Fig. 2) is a highly specific step, the translocation mechanism through 22 stranded beta-barrel TBDTs is likely to be applicable to many other systems sharing similar protein folds (Fig. S6). The three in vitro cross-links we obtained were also observed in vivo as well as two additional cross-links (ColB M19X and G81X with FepA T58 and A214), which we further mapped by LC-MS/ MS (Fig. S3B, Fig. S4D and E). The additional two cross-links did not form in the absence of the energy-transferring protein TonB (Fig. S3C).

The TonB box of TBDTs and bacteriocins is a conserved pentapeptide sequence essential for interaction with TonB (28). Two TonB boxes participate in the ColB translocation process: one on colicin itself and the other on its OM receptor, FepA $(16,29)$. We examined the ability of the in vivo observed ColB 81-FepA 214 cross-link to form as a function of both the FepA and ColB TonB boxes. The ColB 81-FepA 214 cross-link did 


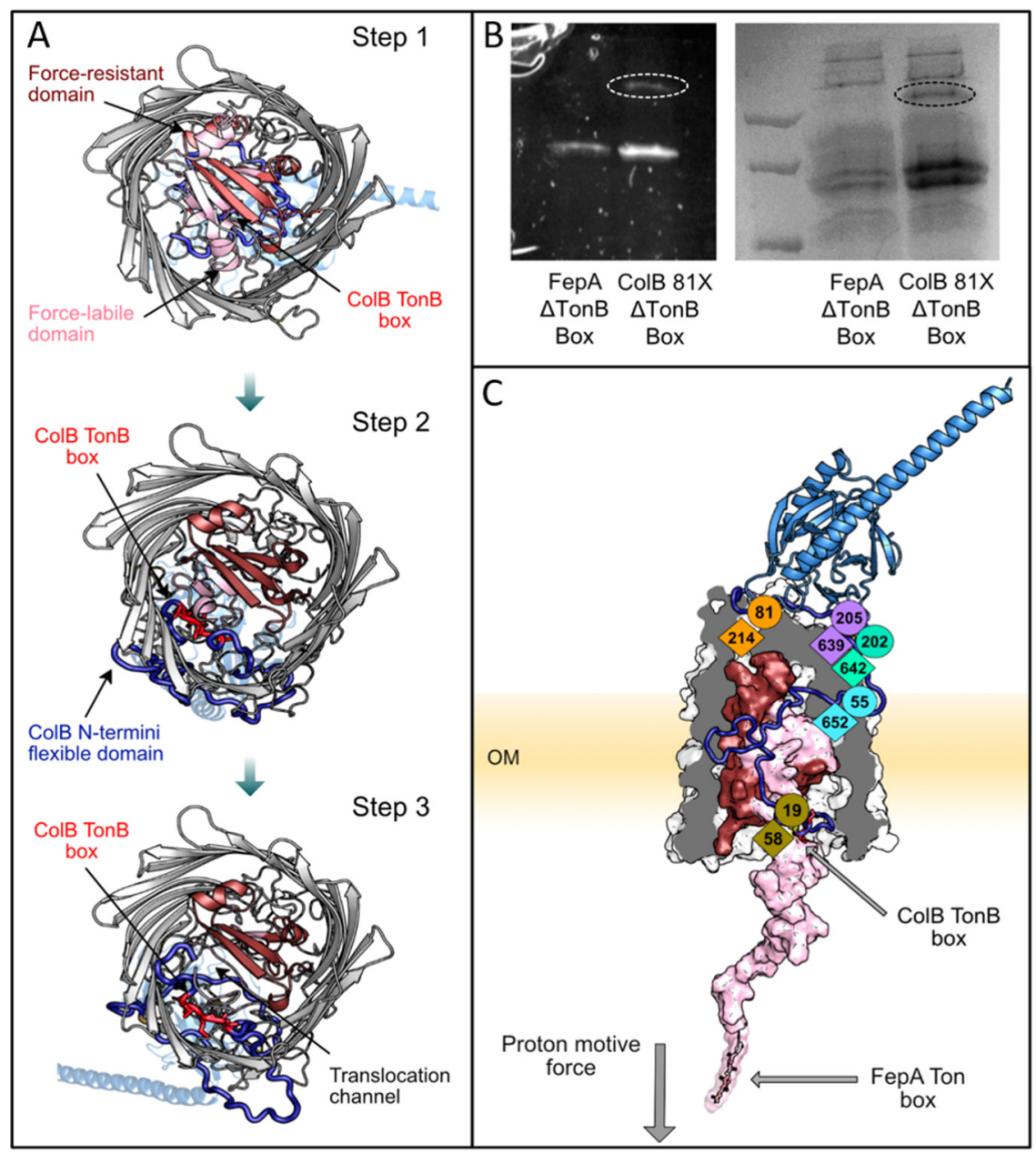

FIG 3 Partially unstructured ColB-RT 55-residue N-terminal end occupies the gap generated by the active unfolding of the FepA N-terminal half plug domain. (A) A bottom-to-top view of the hypothesized translocation pathway (stage 3) created with Rosetta by pulling the FepA N terminus into the cell. Step 1, SC complex is formed and the force-labile half-plug domain (light pink) begins to unfold. Step 2, the force-labile half-plug is partially unfolded, which allows the ColB N-terminal loop (blue) to occupy the void created by the absence of the plug domain. Step 3, the unfolding of the FepA half-plug domain creates a channel for the ColB N-terminal loop to enter. (B) The ability of ColB-81X GFP to cross-link in vivo as a function of both ColB and FepA TonB boxes. GFP fluorescence (right) and Coomassie blue stain (left) are shown. Cross-linked band is circled. (C) The top-scoring model portraying the translocation state (step 3). In this final-stage model, all the cross-link constraints are satisfied and the model is energetically favorable over other states (see Fig. S7 for energy calculations).

not form in the absence of the FepA TonB box, but it still formed in the absence of the ColB TonB box (Fig. 3B). Hence, as both TonB boxes are essential for full colicin translocation, the ColB 81-FepA 214 cross-link appears to capture a stable intermediate translocation step. These experiments were not performed on the second in vivo-identified cross-link ColB 19-FepA 58, as ColB 19 is already part of the ColB TonB box.

To investigate the structures during the dynamic translocation process, we applied Rosetta to simulate the unfolding of the N-terminal half (residues 1 to 74 ) of the globular FepA plug domain, as previously demonstrated for BtuB (30). We simulated the ColB-FepA translocation process starting with the computed SC structure (Fig. $2 \mathrm{C}$ ) and using the in vivo-identified cross-links as guides to generate three intermediate structures in 4- $\AA$ increments (Fig. $3 \mathrm{~A}$ and $\mathrm{C}$ ). The simulated structures suggest that the translocating $\mathrm{N}$-terminal ColB tail (residues 1 to 55 ) occupies the cavity generated by the FepA half plug removal with the ColB TonB box now positioned in place of the former FepA TonB box (Fig. 3A and C). 


\section{DISCUSSION}

The OM of Gram-negative bacteria excludes several classes of antibiotics (31). As a means of subverting this impermeability, Trojan horse antibiotics rely on conjugating antibiotic moieties to siderophores that are actively imported into cells via TBDTs (32, 33). Here, we show, using fluorescence microscopy, that the FepA-specific bacteriocin ColB can similarly transport large cargo molecules into $E$. coli under the force of the PMF (Fig. 1). We also elucidate the mechanism by which ColB binds to FepA and uses the TBDT to translocate across the OM.

ColB was one of the earliest colicins to be identified (34), yet how this bacteriocin, and its close homologue CoID, recognize FepA has been unclear until now. Using photoactivated cross-linking combined with Rosetta-based simulations, we show that association involves at least two steps in which an initial encounter complex is formed that then rearranges. The conformational change involves the flexible $\mathrm{N}$-terminal end of the colicin (residues 1 to 55) moving by up to $62 \AA$ to form the final stable complex. An important consequence of these conformational changes is that they poise the ColB Ton box close to the channel that subsequently appears during PMF-mediated activation of the TBDT by TonB in the inner membrane. While previous studies have demonstrated that the Ton boxes of both ColB and FepA are important for import $(16,29)$, they do not report on the sequence of events where they are deployed. In vivo crosslinking data reveal that the cross-link between ColB-RT G81X and FepA A214 requires the FepA Ton box but not that of ColB (Fig. 3B and Fig. S3B and S4E), consistent with this cross-link reporting on activation of the TBDT by the PMF. The involvement of the ColB Ton box must be subsequent to this, as has been shown for the import of pyocin S2 through its TonB-dependent transporter FpvAl in Pseudomonas aeruginosa (21).

Past chemical modification data have presented a contradictory picture as to whether ColB translocates across the E. coli OM by direct transfer through FepA $(16,35$, 36). Transport of ColB through FepA would require at least partial unplugging of its central pore. Unplugging of a TBDT to enable uptake of a ligand has been demonstrated by atomic force microscopy for the vitamin $B_{12}$ transporter BtuB. The $\mathrm{N}$-terminal globular plug domain of BtuB is composed of two mechanically independent half-plug domains. The N-terminal half, which lies proximal to the Ton box, is more amenable to forced unfolding than the C-terminal half (30). We therefore simulated the unfolding of the N-terminal half-plug of FepA by analogy with that of BtuB (30). The computed model (Fig. 2C) emphasizes the importance of the two independent encounters with the energy-transferring protein TonB. The first receptor-mediated encounter allows the translocation of the ColB TonB box to the periplasm (Fig. 3C), while the second activates colicin translocation into the cell. The computed model also suggests that the 55-residue N-terminal end of the translocating colicin mimics the unfolded receptor half-plug and, indeed, replaces the receptor's TonB box with that from colicin (Fig. $3 \mathrm{~A}$ and $\mathrm{C}$ ).

In summary, the OM translocation of ColB is a highly dynamic process involving two association steps followed by two TonB-dependent events. Our simulations also suggest that colicin mimics the part of the FepA half-plug that is removed during import, thereby presenting its own Ton box to the periplasm. The translocation mechanism likely also applies to ColD, which binds FepA through a similar receptor-binding domain and is Ton dependent (37). The ability of bacteriocin-DNA conjugates to piggy-back the colicin into the cell opens a range of possibilities to utilize bacteriocins for bypassing the Gram-negative bacterial OM. This includes development of novel antibiotic delivery strategies and even genomic manipulations.

\section{MATERIALS AND METHODS}

Protein expression and purification. All colicin constructs were conjugated to a $6 \times \mathrm{His}$ tail at their $\mathrm{C}$ terminus and cloned at the second multiple cloning site of the pACYCDuet-1 (Novagen) plasmid, where they were expressed under a T7 promoter. The plasmids were transformed into BL21(DE3) E. coli cells. Transformed cells were grown at $37^{\circ} \mathrm{C}$ in lysogeny broth (LB), pH 7.2, while shaking at $180 \mathrm{rpm}$ to an optical density at $600 \mathrm{~nm}\left(\mathrm{OD}_{600}\right)$ of $\sim 0.6$, at which point $1 \mathrm{mM}$ isopropyl-D-thiogalactopyranoside (IPTG) was added and the temperature was reduced to $20^{\circ} \mathrm{C}$ for an overnight incubation. Proteinexpressing cells were resuspended in $20 \mathrm{mM}$ Tris, pH 7.5, 0.5 M NaCl, $5 \mathrm{mM}$ imidazole and sonicated 
(70\%, 1.5 min, 3 s on, 7 s off; Sonicator 4000). The sonicated cell extract was spun down and the supernatant was incubated with His-binding resin (69670-5; Merck) for 10 to $30 \mathrm{~min}$ at room temperature. The $\mathrm{Ni}$-resin and the bound protein were then gently $(1,000 \times g)$ spun down, washed three times, and resuspended in the same buffer containing $0.5 \mathrm{M}$ imidazole that allowed protein elution. The protein was dialyzed to phosphate-buffered saline (PBS) at $4^{\circ} \mathrm{C}$ overnight. FepA was expressed on a pBAD/Myc-HisB (Novagen) plasmid transformed into either BI21(DE3) or BW25113 $\Delta$ FepA(JW5086-3) E. coli cells. FepA has been expressed similarly to the colicin proteins, except for the LB growing medium pH being 6.12 and protein expression induction with $0.15 \%$ (wt/vol) L-arabinose. The FepA-containing OM fraction was purified as previously described for OmpF (38). Protein concentrations were determined through absorbance at $280 \mathrm{~nm}$ using a sequence-based extinction coefficient.

Fluorescent labeling of ColB-RT. Colicins were conjugated to Alexa Fluor 488 or 15 b DNA-Alexa Fluor 488 by maleimide reactions as described in Kleanthous et al. (39), with some adaptions: the purified protein was incubated with $10 \mathrm{mM}$ dithiothreitol (DTT) for $1 \mathrm{~h}$ at room temperature (or overnight at $4^{\circ} \mathrm{C}$ ) and was then run through a desalting column (5 ml HiTrap; buffer of $25 \mathrm{mM} \mathrm{Tris,} \mathrm{pH} \mathrm{7.5,} 100 \mathrm{mM}$ $\mathrm{NaCl}$ ) and immediately incubated with a $1.1 \times$ or $3 \times$ ratio of maleimide DNA Alexa Fluor 488 conjugates (generated by Eurogentech) or maleimide Alexa 488, respectively, for $1 \mathrm{~h}$ at room temperature. The reaction was terminated by the addition of $5 \mathrm{mM}$ DTT. The protein was desalted again, retrieved by Ni-beads as in the previous section, and dialyzed to PBS. The efficiency of the fluorescent conjugations was determined by absorbance measurements on a Jasco UV/VIS V-550. The protein-DNA conjugation sensitivity to DNase and trypsin treatments was analyzed on 15\% SDS-PAGE gels.

Microscopy. The following E. coli strains were used for microscopy: MG1655 (wild type), BW25113 (wild type), and BW25113 ( $\triangle$ FepA). A day prior to microscopy, single colonies of each strain were used to inoculate $10 \mathrm{ml} \mathrm{LB}$ and grown overnight at $37^{\circ} \mathrm{C}$. Samples of $200 \mu \mathrm{l}$ of each culture were transferred into $10 \mathrm{ml} \mathrm{M9-glucose} \mathrm{(2} \mathrm{mM} \mathrm{MgSO}, 0.1 \mathrm{mM} \mathrm{CaCl}_{2}, 0.4 \%$ [wt/vol] D-glucose) and grown at $37^{\circ} \mathrm{C}$ for $2.5 \mathrm{~h}$ until an $\mathrm{OD}_{600}$ of $\sim 0.35$, at which point $34 \mu \mathrm{g} / \mathrm{ml}$ chloramphenicol was added to stop further cellular division. Forty-five minutes later the culture was aliquoted to 1-ml treatment tubes to which $100 \mathrm{nM}$ fluorescent protein label was added and incubated with the culture for $1 \mathrm{~h}$ at $37^{\circ} \mathrm{C}$. The cells were then washed three times in PBS. All different treatments (simple labeling/trypsin-treated cells/spheroplasted cells) were resuspended in $10 \mu \mathrm{l}$ PBS (with $0.5 \mathrm{M}$ sucrose and $20 \mathrm{mM} \mathrm{MgCl}$ for spheroplasted cells), of which $5 \mu \mathrm{l}$ was placed onto $1 \%$ agarose pads (containing $0.5 \mathrm{M}$ sucrose and $20 \mathrm{mM} \mathrm{MgCl}_{2}$ for the spheroplasts). All imaging was conducted on the ONI Nanoimager S. A 473-nm laser was used at $20 \%$ laser power to visualize cells labeled with Alexa 488. For each field of view, 10 frames were collected at an exposure of $100 \mathrm{~ms}$. For data analysis purposes for each field of view, the 10 frames collected were averaged, and the fluorescent intensity of the cells and of their surrounding background was measured. Data analysis was performed by ImageJ software. Thirty cells and adjusted background intensities were analyzed for each treatment at each experiment. Each experiment was repeated 2 to 4 times on newly grown labeled and treated cells.

Trypsin and spheroplast treatment for microscopy. Trypsin treatment was applied on cells that had undergone the labeling procedure described in the previous section to determine whether the fluorescent signal translocated into the cells. The cells were incubated with $1 \mathrm{mg} / \mathrm{ml}$ trypsin in $\mathrm{PBS}, \mathrm{pH} 7.8$, for $1 \mathrm{~h}$ at $37^{\circ} \mathrm{C}$. The trypsin was then washed two times in PBS. To determine whether the fluorescent signal translocated into the cytoplasm, the cells were further spheroplasted. The trypsin-treated cells were resuspended in $0.8 \mathrm{M}$ Tris, $\mathrm{pH} 8,0.5 \mathrm{M}$ sucrose, $1 \mathrm{mg} / \mathrm{ml} \mathrm{lysozyme,} 2 \mathrm{mM}$ EDTA for $30 \mathrm{~min}$ at room temperature, and $0.1 \mathrm{mg} / \mathrm{ml}$ trypsin was then added and incubated with the mixture for an additional $30 \mathrm{~min}$. The cells where then washed in PBS, $0.5 \mathrm{M}$ sucrose, $20 \mathrm{mM} \mathrm{MgCl}$.

Native-state electrospray ionization mass spectrometry. Sixty milligrams of ColB-RT (341 amino acids [aa]) were added to a 5-liter culture of BW25113( $\triangle$ FepA) cells overexpressing FepA from a pBAD/ Myc-HisB (Novagen) plasmid. The complex was purified by following the protocol previously described for OmpF (38). A 5-ml HiTrap desalting column (GE Healthcare) was used to exchange the complex buffer into $100 \mathrm{mM}$ ammonium acetate, $1 \%$ (wt/vol) $n$-octyl- $\beta$-D-glucopyranoside ( $\beta$-OG), pH 6.9. Mass spectrometry measurements were made from a static nanospray emitter using gold-coated capillaries prepared in-house (40) on a quadrupole time-of-flight mass spectrometer (Micromass) modified for high mass transmission. Liberation of the protein complex from $\beta$-OG detergent required energetic instrument parameters, and the low $\mathrm{m} / \mathrm{z}$ region of spectra was dominated by detergent clusters. Operating conditions used include capillary voltage of 1,800 V, sample cone of $200 \mathrm{~V}$, extractor of $10 \mathrm{~V}$, collision cell energy of 140 to $200 \mathrm{~V}$, source backing pressure of $5.92 \times 10^{-3} \mathrm{mbar}$, and argon collision cell pressure of 3.5 to $5 \mathrm{MPa}$.

Cross-linking. The cross-linking procedure was similar to that of White et al. (21). In short, $p B P A$ mutations were introduced at 21 different positions of ColB-RT (341 aa) green fluorescent protein (GFP). For in vitro cross-linking, $1 \mu \mathrm{M} p B P A$ containing colicin was incubated with $1 \mathrm{ml}$ of an OM protein fraction (in PBS, pH 6.5, 5 mM EDTA, 2\% $\beta$-OG) extracted from BW25113 FepA knockout cells over expressing FepA containing $\sim 1 \mu \mathrm{M}$ FepA and exposed to UV light (365 nm) for $1 \mathrm{~h}$ at $4^{\circ} \mathrm{C}$. The colicin and bound/cross-linked FepA were then extracted by EDTA-resistant Ni-beads cOmplete (Merck). For in vivo cross-linking, the colicin was incubated with $800 \mathrm{ml}$ cells that overexpressed FepA through an overnight incubation at $20^{\circ} \mathrm{C}$. The $p B P A$ containing colicin was added to the LB medium $(\mathrm{pH} 6.12)$ and incubated for $90 \mathrm{~min}$ at $37^{\circ} \mathrm{C}$ while shaking. The cells were then spun down $\left(3,000 \times g, 20 \mathrm{~min}, 4^{\circ} \mathrm{C}\right)$, colicin excess was washed with $50 \mathrm{ml}$ PBS, the cells were resuspended in $10 \mathrm{ml}$ PBS and exposed to UV light (365 nm) for $1 \mathrm{~h}$ at $4^{\circ} \mathrm{C}$. The cells were then resuspended in $10 \mathrm{mM}$ Tris, $\mathrm{pH} 8,0.25 \%$ lithium diiodosalicylic acid (LIS), $2 \%$ Triton X-100, sonicated, the cell debris were spun down $\left(10,000 \times g, 10 \mathrm{~min}, 4^{\circ} \mathrm{C}\right)$, and the supernatant ultracentrifuged $\left(200,000 \times g, 45 \mathrm{~min}, 4^{\circ} \mathrm{C}\right)$. The pellet was resuspended in $\mathrm{PBS}, \mathrm{pH} 6.5,5 \mathrm{mM}$ 
EDTA, 2\% $\beta$-OG, ultracentrifuged again, and the colicin with its bound/cross-linked proteins was extracted by EDTA-resistant Ni-bead cOmplete (Merck). The extracted proteins were run on 12\% SDSPAGE gels, and GFP fluorescent bands of adequate size were analyzed by LC-MS/MS for cross-linking mapping.

LC-MS/MS cross-linking analysis. Peptides were separated on an EASY-nLC 1000 ultrahigh-performance liquid chromatography (UHPLC) system (Proxeon) and electrosprayed directly into a $Q$ Exactive mass spectrometer (Thermo Fisher). Peptides were trapped on a $C_{18}$ PepMap100 precolumn (300- $\mu \mathrm{m}$ inner diameter by $5 \mathrm{~mm}, 100$-Å pore size; Thermo Fisher) using solvent A $(0.1 \%$ [vol/vol] formic acid in water) at $500 \times 10^{5} \mathrm{~Pa}$ and then separated on an in-house-packed analytical column (50 cm by 75- $\mu \mathrm{m}$ inner diameter packed with ReproSil-Pur $120 \mathrm{C}_{18}-\mathrm{AQ}, 1.9 \mu \mathrm{m}, 120 \AA$ pore size; Maisch $\mathrm{GmbH}$ ) with a linear gradient from $10 \%$ to $55 \%$ (vol/vol) solvent B $(0.1 \%$ [vol/vol] formic acid in acetonitrile) in $45 \mathrm{~min}$ at $200 \mathrm{nl} / \mathrm{min}$. Full-scan MS spectra were acquired in the Orbitrap (scan range, 350 to 2,000 m/z; resolution, 70,000; automatic gain control target, 3e6; maximum injection time, $100 \mathrm{~ms}$ ). After the MS scans, the 10 most intense peaks were selected for higher-energy collisional dissociation (HCD) fragmentation at $30 \%$ of normalized collision energy. HCD spectra were also acquired in the Orbitrap (resolution, 17,500; automatic gain control target, 5e4; maximum injection time, $120 \mathrm{~ms}$ ) with first fixed mass at $100 \mathrm{~m} / \mathrm{z}$. Charge states $1+$ and $2+$ were excluded from HCD fragmentation. MS data were searched using the plink software (41). The database contained the target proteins and common contaminants. Search parameters were the following: maximum number of missed cleavages, 2; fixed modification, carbamidomethyl-Cys; variable modification 1, oxidation-Met; variable modification 2, Glu to pyro-Glu. Cross-linking from D to $\mathrm{K}, \mathrm{S}, \mathrm{T}$, or $\mathrm{N}$ terminus was considered. Data were initially filtered to a false discovery rate (FDR) of $1 \%$. Cross-links were further filtered/inspected with specific emphasis on fragmentation patterns.

Structural modeling. (i) Computational modeling of the FepA-ColB interaction: structure preparation. The crystal structures of ColB (1RH1 [24]) and FepA (1FEP [18]) were used as starting templates for the computational modeling. Because the crystal structures were missing key loops needed to effectively propagate backbone motions, we added these loops (residues 31 to 44 on ColB and 323 to 335 and 384 to 40 on FepA) using SWISS MODELLER (42). To eliminate energetically unfavorable side chain or backbone clashes, we then relaxed the structures using constraints to the native crystal coordinates using RosettaRelax (43).

(ii) Stage 1: modeling the semirigid EC. We determined putative local binding conformations by first performing rigid-body global docking using Rosetta's ReplicaDock2 protocol (built upon prior work on temperature and Hamiltonian replica exchange Monte Carlo approaches [44, 45]) and clustering the lowest-energy docked structures. Starting from each low-energy structure, we refine the structures in a local binding region by using our RosettaDock4.0 (46) protocol that adaptively swaps receptor and ligand conformations from a pregenerated ensemble of structures. We diversify the backbone conformations in the ensemble by using (i) ReplicaDock 2.0, (ii) Rosetta Relax (43), and (iii) Rosetta Backrub (47). Local docking generates $\sim 6,000$ decoys, which are scored based on their interface energies, defined as the energy difference between the total energy of the complex and the total energy of the monomers in isolation (see the supplemental material for details and command lines).

(iii) Stage 2: modeling the SC, allowing backbone flexibility. To explore the possibility of the ColB flexible N-terminal domain (residues 1 to 55) interacting explicitly with FepA, we used the Rosetta FloppyTail (48) algorithm, which allows modest sampling of backbone degrees of freedom following a two-stage approach. First, in the low-resolution stage, side chains are represented by a centroid atom and the backbone conformational space is extensively sampled. In the high-resolution stage, all side chain atoms then are returned to refine the structures. We generated $\sim 5,000$ hypothetical decoys starting from the encounter complex obtained in stage 1 (EC). The 5,000 perturbation cycles and 1,000 refinement cycles were used for each decoy. To direct the MC sampling of the FloppyTail algorithm toward possible interacting regions, atom-pair constraints based on the experimental (in vitro) cross-linking residues guided the search. These constraints were calculated based on a harmonic potential with a mean of $6 \AA$ and a standard deviation set to $0.25 \AA$ between the $\mathrm{C} \alpha$ atoms of the candidate residues. Each output decoy was further relaxed to remove unfavorable clashes, and the 100 top-scoring models were then docked using RosettaDock4.0 (46) using a fixed backbone. Translational and rotational moves were performed on the top models to generate $\sim 5,000$ docking decoys. To confirm the feasibility of these decoys, we evaluated the interface energies and compared the energy landscape of decoys in stage 2 with the prior decoys obtained in stage 1 (Fig. 1C).

(iv) Stage 3: prediction of the translocation pathway applying in vivo cross-linking data. Following the partial unfolding of the plug domain in the related TonB-BtuB system (30), we allowed backbone movement in the FepA 75-residue half-plug domain (residues 1 to 75) and the ColB flexible Nterminal domain (residues 1 to 43). Since simulating the dynamic unfolding of FepA half-plug with simultaneous translocation of the ColB via the barrel protein would be intensely demanding computationally, we instead create models to represent three steps along the dynamic pathway of the unfolding translocation process. A figure showing the workflow with intermediate snapshots and complete details of each phase of our three-part model creation are given in the supplemental computational methods. Briefly, to create each structure along the pathway, we (i) displace the FepA half-plug (residues 1 to 75 ) using Rosetta FloppyTail to pull the terminus out by 4,8 , and $12 \AA$, respectively, to begin making each of the three structural steps in the pathway; (ii) translocate the ColB N-terminal domain (residues 1 to 43) using both in vitro and in vivo cross-linking constraints with Rosetta FloppyTail; and (iii) refine both FepA and ColB conformation and rigid-body displacement using RosettaDock with a flexible FepA halfplug and ColB N-terminal domain. During stages 1 and 2, backbone motions in FloppyTail are 
propagated toward the closest terminus, but in stage 3, ColB backbone perturbations during docking are propagated back toward the bulk of ColB to facilitate it finding the optimal rigid-body displacement while the $\mathrm{N}$-terminal domain is translocating. Finally, we calculate interface scores to reveal the favorability relative to conformations of other models presented in this paper along the hypothesized unfolding-translocation pathway (Fig. S7).

\author{
SUPPLEMENTAL MATERIAL \\ Supplemental material is available online only. \\ MOVIE S1, MPG file, 1.3 MB \\ TEXT S1, DOCX file, 0.6 MB. \\ FIG S1, TIF file, 0.2 MB. \\ FIG S2, TIF file, $0.4 \mathrm{MB}$. \\ FIG S3, PDF file, $2.5 \mathrm{MB}$. \\ FIG S4, PDF file, 2.4 MB. \\ FIG S5, TIF file, 0.7 MB. \\ FIG S6, PDF file, $0.4 \mathrm{MB}$. \\ FIG S7, TIF file, $0.1 \mathrm{MB}$.
}

\title{
ACKNOWLEDGMENTS
}

The experimental work was funded by BBSRC grant BB/P009948/1. A.H. and J.J.G. were funded by the U.S. National Institutes of Health grant R01-GM078221.

\section{REFERENCES}

1. Hibbing ME, Fuqua C, Parsek MR, Peterson SB. 2010. Bacterial competition: surviving and thriving in the microbial jungle. Nat Rev Microbiol 8 : 15-25. https://doi.org/10.1038/nrmicro2259.

2. Khan A, Singh P, Srivastava A. 2018. Synthesis, nature and utility of universal iron chelator-Siderophore: a review. Microbiol Res 212-213:103-111. https://doi.org/10.1016/j.micres.2017.10.012.

3. Aoki SK, Diner EJ, de Roodenbeke CT, Burgess BR, Poole SJ, Braaten BA, Jones AM, Webb JS, Hayes CS, Cotter PA, Low DA. 2010. A widespread family of polymorphic contact-dependent toxin delivery systems in bacteria. Nature 468:439-442. https://doi.org/10.1038/nature09490.

4. Montville TJ, Bruno MEC. 1994. Evidence that dissipation of proton motive force is a common mechanism of action for bacteriocins and other antimicrobial proteins. Int J Food Microbiol 24:53-74. https://doi.org/10 1016/0168-1605(94)90106-6.

5. Papadakos G, Wojdyla JA, Kleanthous C. 2012. Nuclease colicins and their immunity proteins. Q Rev Biophys 45:57-103. https://doi.org/10.1017/ S0033583511000114.

6. Green ER, Mecsas J. 2016. Bacterial secretion systems: an overview. Microbiol Spectr 4:10.1128/microbiolspec.VMBF-0012-2015. https://doi.org/10 .1128/microbiolspec.VMBF-0012-2015.

7. Simons A, Alhanout K, Duval RE. 2020. Bacteriocins, antimicrobial peptides from bacterial origin: overview of their biology and their impact against multidrug-resistant bacteria. Microorganisms 8:639. https://doi .org/10.3390/microorganisms8050639.

8. Cascales E, Buchanan SK, Duché D, Kleanthous C, Lloubès R, Postle K, Riley M, Slatin S, Cavard D. 2007. Colicin biology. Microbiol Mol Biol Rev 71: 158-229. https://doi.org/10.1128/MMBR.00036-06.

9. Kleanthous C. 2010. Swimming against the tide: progress and challenges in our understanding of colicin translocation. Nat Rev Microbiol 8: 843-848. https://doi.org/10.1038/nrmicro2454.

10. Masi M, Réfregiers M, Pos KM, Pagès J-M. 2017. Mechanisms of envelope permeability and antibiotic influx and efflux in Gram-negative bacteria. Nat Microbiol 2:17001. https://doi.org/10.1038/nmicrobiol.2017.1.

11. Braun V, Pilsl H, Gross P. 1994. Colicins: structures, modes of action, transfer through membranes, and evolution. Arch Microbiol 161:199-206. https://doi.org/10.1007/BF00248693.

12. Egan AJF. 2018. Bacterial outer membrane constriction. Mol Microbiol 107:676-687. https://doi.org/10.1111/mmi.13908.

13. Szczepaniak J, Press C, Kleanthous C. 2020. The multifarious roles of TolPal in Gram-negative bacteria. FEMS Microbiol Rev 44:490-506. https:// doi.org/10.1093/femsre/fuaa018.

14. Ratliff AC, Buchanan SK, Celia H. 2021. Ton motor complexes. Curr Opin Struct Biol 67:95-100. https://doi.org/10.1016/j.sbi.2020.09.014.
15. Arima K, Katoh Y, Beppu T. 1968. Studies on colicin B: mode of action and new extraction method from cells. Agric Biol Chem 32:170-177. https:// doi.org/10.1271/bbb1961.32.170.

16. Devanathan S, Postle K. 2007. Studies on colicin B translocation: FepA is gated by TonB. Mol Microbiol 65:441-453. https://doi.org/10.1111/j.1365 -2958.2007.05808.x.

17. Pressler U, Braun V, Wittmann-Liebold B, Benz R. 1986. Structural and functional properties of colicin B. J Biol Chem 261:2654-2659. https://doi .org/10.1016/S0021-9258(17)35837-4.

18. Buchanan SK, Smith BS, Venkatramani L, Xia D, Esser L, Palnitkar M, Chakraborty R, van der Helm D, Deisenhofer J. 1999. Crystal structure of the outer membrane active transporter FepA from Escherichia coli. Nat Struct Biol 6:56-63. https://doi.org/10.1038/4931.

19. Dhar R, Slusky JS. 2021. Outer membrane protein evolution. Curr Opin Struct Biol 68:122-128. https://doi.org/10.1016/j.sbi.2021.01.002.

20. Franklin MW, Nepomnyachyi S, Feehan R, Ben-Tal N, Kolodny R, Slusky JS. 2018. Evolutionary pathways of repeat protein topology in bacterial outer membrane proteins. Elife 7:e40308. https://doi.org/10.7554/eLife.40308.

21. White $P$, Joshi A, Rassam P, Housden NG, Kaminska R, Goult JD, Redfield C, McCaughey LC, Walker D, Mohammed S, Kleanthous C. 2017. Exploitation of an iron transporter for bacterial protein antibiotic import. Proc Natl Acad Sci U S A 114:12051-12056. https://doi.org/10.1073/pnas .1713741114

22. Behrens HM, Lowe ED, Gault J, Housden NG, Kaminska R, Weber TM, Thompson CMA, Mislin GLA, Schalk IJ, Walker D, Robinson CV, Kleanthous C. 2020. Pyocin S5 import into Pseudomonas aeruginosa reveals a generic mode of bacteriocin transport. mBio 11:e03230-19. https://doi.org/10 .1128/mBio.03230-19.

23. Harmalkar A, Gray JJ. 2021. Advances to tackle backbone flexibility in protein docking. Curr Opin Struct Biol 67:178-186. https://doi.org/10.1016/j .sbi.2020.11.011.

24. Hilsenbeck JL, Park H, Chen G, Youn B, Postle K, Kang C. 2004. Crystal structure of the cytotoxic bacterial protein colicin $B$ at $2.5 \AA$ A resolution. Mol Microbiol 51:711-720. https://doi.org/10.1111/j.1365-2958.2003.03884.x.

25. Cheng Y-S, Shi Z, Doudeva LG, Yang W-Z, Chak K-F, Yuan HS. 2006. Highresolution crystal structure of a truncated ColE7 translocation domain: implications for colicin transport across membranes. J Mol Biol 356:22-31 . https://doi.org/10.1016/j.jmb.2005.11.056.

26. Dormán G, Nakamura H, Pulsipher A, Prestwich GD. 2016. The life of pi star: exploring the exciting and forbidden worlds of the benzophenone photophore. Chem Rev 116:15284-15398. https://doi.org/10.1021/acs .chemrev.6b00342. 
27. Leman JK, Weitzner BD, Lewis SM, Adolf-Bryfogle J, Alam N, Alford RF, Aprahamian M, Baker D, Barlow KA, Barth P, Basanta B, Bender BJ, Blacklock K, Bonet J, Boyken SE, Bradley P, Bystroff C, Conway P, Cooper S, Correia BE, Coventry B, Das R, De Jong RM, DiMaio F, Dsilva L, Dunbrack R, Ford AS, Frenz B, Fu DY, Geniesse C, Goldschmidt L, Gowthaman R, Gray JJ, Gront D, Guffy S, Horowitz S, Huang P-S, Huber T, Jacobs TM, Jeliazkov JR, Johnson DK, Kappel K, Karanicolas J, Khakzad H, Khar KR, Khare SD, Khatib F, Khramushin A, King IC, Kleffner R, et al. 2020. Macromolecular modeling and design in Rosetta: recent methods and frameworks. Nat Methods 17:665-680. https://doi.org/10.1038/s41592-020-0848-2.

28. Schramm E, Mende J, Braun V, Kamp RM. 1987. Nucleotide sequence of the colicin B activity gene cba: consensus pentapeptide among TonB-dependent colicins and receptors. J Bacteriol 169:3350-3357. https://doi .org/10.1128/jb.169.7.3350-3357.1987.

29. Mende J, Braun V. 1990. Import-defective colicin B derivatives mutated in the TonB box. Mol Microbiol 4:1523-1533. https://doi.org/10.1111/j.1365 -2958.1990.tb02063.x.

30. Hickman SJ, Cooper REM, Bellucci L, Paci E, Brockwell DJ. 2017. Gating of TonB-dependent transporters by substrate-specific forced remodelling. Nat Commun 8:14804. https://doi.org/10.1038/ncomms14804.

31. Ghai I, Ghai S. 2018. Understanding antibiotic resistance via outer membrane permeability. Infect Drug Resist 11:523-530. https://doi.org/10 .2147/IDR.S156995.

32. Górska A, Sloderbach A, Marszałł MP. 2014. Siderophore-drug complexes: potential medicinal applications of the "Trojan horse" strategy. Trends Pharmacol Sci 35:442-449. https://doi.org/10.1016/j.tips.2014.06.007.

33. Kong H, Cheng W, Wei H, Yuan Y, Yang Z, Zhang X. 2019. An overview of recent progress in siderophore-antibiotic conjugates. Eur J Med Chem 182:111615. https://doi.org/10.1016/j.ejmech.2019.111615.

34. Fredericq P. 1950. Research on the characteristics and distribution of strains producing colicine B. C R Seances Soc Biol Fil 144:1287-1289.

35. Ma L, Kaserer W, Annamalai R, Scott DC, Jin B, Jiang X, Xiao Q, Maymani H, Massis LM, Ferreira LCS, Newton SMC, Klebba PE. 2007. Evidence of balland-chain transport of ferric enterobactin through FepA. J Biol Chem 282: 397-406. https://doi.org/10.1074/jbc.M605333200.

36. Smallwood CR, Marco AG, Xiao Q, Trinh V, Newton SMC, Klebba PE. 2009. Fluoresceination of FepA during colicin B killing: effects of temperature, toxin and TonB. Mol Microbiol 72:1171-1180. https://doi.org/10.1111/j .1365-2958.2009.06715.x.

37. Rabsch W, Ma L, Wiley G, Najar FZ, Kaserer W, Schuerch DW, Klebba JE, Roe BA, Laverde Gomez JA, Schallmey M, Newton SMC, Klebba PE. 2007.
FepA-and TonB-dependent bacteriophage $\mathrm{H} 8$ : receptor binding and genomic sequence. J Bacteriol 189:5658-5674. https://doi.org/10.1128/JB .00437-07.

38. Housden NG, Wojdyla JA, Korczynska J, Grishkovskaya I, Kirkpatrick N, Brzozowski AM, Kleanthous C. 2010. Directed epitope delivery across the Escherichia coli outer membrane through the porin OmpF. Proc Natl Acad Sci U S A 107:21412-21417. https://doi.org/10.1073/pnas.1010780107.

39. Kleanthous C, Rassam P, Baumann CG. 2015. Protein-protein interactions and the spatiotemporal dynamics of bacterial outer membrane proteins. Curr Opin Struct Biol 35:109-115. https://doi.org/10.1016/j.sbi.2015.10.007.

40. Hernandez H, Robinson CV. 2007. Determining the stoichiometry and interactions of macromolecular assemblies from mass spectrometry. Nat Protoc 2:715-726. https://doi.org/10.1038/nprot.2007.73.

41. Yang B, Wu Y-J, Zhu M, Fan S-B, Lin J, Zhang K, Li S, Chi H, Li Y-X, Chen HF, Luo S-K, Ding Y-H, Wang L-H, Hao Z, Xiu L-Y, Chen S, Ye K, He S-M, Dong M-Q. 2012. Identification of cross-linked peptides from complex samples. Nat Methods 9:904-906. https://doi.org/10.1038/nmeth.2099.

42. Webb B, Sali A. 2016. Comparative protein structure modeling using MODELLER. Curr Protoc Bioinformatics 54:5.6.1-5.6.37. https://doi.org/10 .1002/cpbi.3.

43. Conway P, Tyka MD, DiMaio F, Konerding DE, Baker D. 2014. Relaxation of backbone bond geometry improves protein energy landscape modeling. Protein Sci 23:47-55. https://doi.org/10.1002/pro.2389.

44. Zhang Z, Lange OF. 2013. Replica exchange improves sampling in low-resolution docking stage of RosettaDock. PLoS One 8:e72096. https://doi .org/10.1371/journal.pone.0072096.

45. Zhang Z, Schindler CE, Lange OF, Zacharias M. 2015. Application of enhanced sampling Monte Carlo methods for high-resolution proteinprotein docking in Rosetta. PLoS One 10:e0125941. https://doi.org/10 .1371/journal.pone.0125941.

46. Marze NA, Roy Burman SS, Sheffler W, Gray JJ. 2018. Efficient flexible backbone protein-protein docking for challenging targets. Bioinformatics 34:3461-3469. https://doi.org/10.1093/bioinformatics/bty355.

47. Smith CA, Kortemme T. 2008. Backrub-like backbone simulation recapitulates natural protein conformational variability and improves mutant sidechain prediction. J Mol Biol 380:742-756. https://doi.org/10.1016/j.jmb.2008 .05.023.

48. Kleiger G, Saha A, Lewis S, Kuhlman B, Deshaies RJ. 2009. Rapid E2-E3 assembly and disassembly enable processive ubiquitylation of cullin-RING ubiquitin ligase substrates. Cell 139:957-968. https://doi.org/10.1016/j .cell.2009.10.030. 